\title{
Activación sociocomunitaria: claves para la inclusión y la cohesión social
}

\section{Pablo Ruiz Errea}

Bizitegi

\section{Aitor Aresti}

Bizitegi

\author{
Aitor Ipiña \\ Bizitegi \\ <aipina@bizitegi.org>
}

La evolución de la sociedad y de los servicios sociales ha promovido una inclusión social centrada en el empleo como herramienta de autonomía personal y de participación social. Para dar respuesta al importante grupo de personas excluidas que no encuentran empleo o tienen grandes dificultades, el presente artículo propone hacer del trabajo comunitario un pilar central en el proceso de inclusión social, inclusión que se pretende basar en el fortalecimiento de las relaciones personales en los entornos más cercanos. Asimismo, el artículo profundiza en los conceptos de inclusión activa, activación inclusiva, ciudadanía activa y activación comunitaria, para terminar con la propuesta novedosa de crear un centro de activación sociocomunitaria.

\section{PALABRAS ClaVE:}

Exclusión social, inclusión social, inclusión activa, ciudadanía activa, activación comunitaria. 


\section{Evolución de las organizaciones sociales y de la sociedad}

La realidad social actual nos sitúa frente a la necesidad de desarrollar nuevos planteamientos, nuevos esquemas conceptuales desde los que orientar la inclusión social. Muchas organizaciones sociales en Euskadi llevamos más de tres décadas trabajando con las personas en exclusión, y debemos repensar nuestros esquemas de funcionamiento a la vista de los cambios que se han producido. Si las realidades económicas, tecnológicas, migratorias, medioambientales, en definitiva, si la realidad social global ha cambiado tanto, también lo tienen que hacer nuestras respuestas.

Es por ello por lo que vamos a empezar nuestra aportación con una visión retrospectiva crítica de nuestra evolución como organización, que, entendemos, refleja la evolución general del sector. Desde esa experiencia, planteamos una propuesta concreta de actuación, que se basa en ideas innovadoras, a la vez que rescata claves que se han ido perdiendo en el proceso de institucionalización. Consideramos que nuestro papel, como entidad del tercer sector, es explorar caminos que puedan ir ensanchando el horizonte de los servicios sociales que la Administración preste a toda la ciudadanía.

De los sistemas de protección con los que se ha ido dotando el Estado del bienestar, el de servicios sociales es el más reciente, habiéndose desarrollado principalmente a partir de la década de los ochenta, época también de la creación de Bizitegi. En ese momento, nos encontrábamos con un contexto social en el que la crisis asolaba en especial al País Vasco, donde las cifras del paro rondaban el $25 \%$, terreno abonado para que las situaciones de exclusión crecieran de forma alarmante, como así hicieron. Era una sociedad económicamente más pobre, donde muchos de sus miembros jóvenes sufrían las consecuencias de un consumo de tóxicos que derivaba en problemas de salud, pérdida de redes sociales, empobrecimiento y delincuencia.

En aquel momento, las situaciones de exclusión eran percibidas por la ciudadanía como algo próximo. Era habitual conocer a alguien, más o menos cercano, con un problema de drogadicción. Lo normal era pensar que los seres queridos (especialmente, los más jóvenes) no estaban totalmente libres de riesgo, y los altos índices de paro y las dificultades para encontrar un empleo generaban una sensación compartida de inseguridad.

La cobertura social de las administraciones públicas, sumidas en el inicio del proceso de descentralización, era débil, y la sociedad vivía acostumbrada a unirse y trabajar para buscar soluciones a los problemas de sus miembros. Por todo ello, cualquier iniciativa de este tipo era bien recibida por la ciudadanía. Y así nacieron la mayor parte de los recursos sociales, como resultado de una sociedad civil organizada, con una elevada cohesión interna.
Estos recursos nacieron con un marcado carácter comunitario y se ubicaban en barrios en los que había un alto índice de personas con problemas de exclusión. Los dispositivos eran aceptados en sus entornos, que reconocían los beneficios que aportaban a la vecindad, valorando especialmente su proximidad y flexibilidad. La relación con las personas (no con sus problemas y patologías) se daba en el mismo contexto en el que éstas desarrollaban su vida cotidiana, y perseguía el objetivo de que éstas llegaran a vivir de forma autónoma. Un porcentaje significativo de ellas eran capaces de alcanzar ese objetivo final, a través del refuerzo de la confianza en sí mismas, la abstinencia en el consumo de tóxicos, el inicio y mantenimiento de tratamiento, la recuperación de capacidades o la adquisición de nuevas habilidades, la generación de nuevas redes de apoyo social y el acceso a oportunidades que antes no estaban a su alcance (relacionales, formativas, de empleo).

Comenzamos a hablar de inserción social -término que comenzó a usarse a finales de los setenta en Francia (Subirats, 2004) - entendiéndola, como dice Raya Lozano (2004), como una serie de medidas políticas y movilización de recursos para incorporar al sistema a aquellas personas que, por diferentes causas, no tenían trabajo. El empleo aparecía como el gran objetivo de los planes de inclusión de las personas atendidas en la red de servicios sociales. Su trabajo era capacitarlas para que pudieran conseguir y mantener un empleo. Este enfoque encaja, además, con una sociedad en la que el modelo estándar de inclusión gira alrededor del trabajo remunerado, puesto que éste ofrece acceso a recursos económicos, necesarios para mantener una vida autónoma; oportunidades de participación, por el desarrollo de labores socialmente útiles; y supone una aportación al bien común, a través del pago de impuestos.

Durante las siguientes décadas, todo esto ha ido evolucionando. Las instituciones públicas se han ido fortaleciendo tanto en lo económico, al dotarse de más recursos, como en el marco normativo, generando leyes que han permitido orientarlos en beneficio de la ciudadanía. El sistema de protección social se ha reforzado y las administraciones han ido asumiendo un mayor nivel de responsabilidad y cobertura. Esto ha conllevado un crecimiento de las organizaciones que llevaban años trabajando a favor de los más desfavorecidos, y una profesionalización de aquéllas. Paralelamente, la intervención social ha sufrido grandes progresos técnicos en los que, manteniendo el espíritu fundacional de las organizaciones, se ha superado el voluntarismo inicial. Esta mejora técnica y de cuidados, unida a los avances de la medicina, ha garantizado la supervivencia de muchas personas, que, en la situación anterior, no hubieran podido sobrevivir a las causas de su situación de exclusión social.

Por otro lado, la mejora económica que ha experimentado nuestra sociedad ha provocado 
que se haya invertido el flujo migratorio, y hemos pasado a ser receptores de personas que vienen de otros países. Todo esto ha implicado un cambio en el perfil de las personas que atienden los servicios sociales. Hay muchas más personas venidas de fuera, con disposición y capacidad para trabajar, pero sin la condición de ciudadanos de pleno derecho. Y entre las autóctonas, han ido quedando bajo el manto de los servicios sociales aquellas que tienen mayores dificultades para acceder al mundo laboral y desarrollar una vida autónoma.

Las políticas sociales se han seguido construyendo en torno al objetivo del empleo, y la ciudadanía ha ido asumiendo que es adecuado dedicar recursos para atender a las personas siempre que se haga desde el principio de la reciprocidad, es decir, apoyar a quienes, con el tiempo, van a llegar a ser productivas y van a poder 'devolver' aquello que han recibido. Las organizaciones del tercer sector, alineadas con los sistemas de financiación pública de servicios, hemos construido una red de atención con la autonomía plena y el empleo como objetivos finales.

El balance de todo este desarrollo ha sido, en líneas generales, satisfactorio. Sin embargo, en toda esta dinámica positiva de mejora y crecimiento, no hemos sabido detectar algunos cambios que, paulatinamente, se han ido produciendo, y que han derivado en algunas consecuencias no deseables.

\subsection{Alejamiento de los barrios de origen}

A ojos de la comunidad en la que se encuentran los dispositivos, éstos comenzaron siendo una solución, ya que atendían a personas vecinas, conocidas en su entorno, que tenían dificultades y llamaban a su puerta. En cambio, hoy en día se atiende a individuos anónimos a los que las administraciones públicas expiden un certificado sobre su situación de exclusión y que proceden no se sabe de qué barrio, pueblo o país. Por lo tanto, de cara al vecindario se ha pasado de ser una solución a los problemas del barrio a resultar molestos, porque acuden personas problemáticas que provienen de otros lugares.

Éste ha sido un proceso de cambio lento del que no hemos sido conscientes y que se ha ido produciendo mientras seguíamos centrados en atender a las personas que acudían derivadas a nuestros centros. Se ha ido generando un ambiente cada vez menos receptivo e incluso hostil, donde aquellas comunidades en las que ya había alguno implantado han seguido aceptando estos dispositivos, porque no les han generado mucha molestia, pero los intentos de abrir otros nuevos no han sido bienvenidos.

En un análisis excesivamente superficial, estos recursos se han seguido considerando como comunitarios, porque no se han movido de donde estaban. Y nos hemos sentido víctimas de la falta de comprensión de parte de la ciudadanía, sin haber sabido ver que no estábamos haciendo lo suficiente para seguir aportando valor añadido a los barrios.

\subsection{Búsqueda de objetivos equivocados}

Como ya hemos dicho, el empleo ha sido, durante todos estos años, el principal objetivo de las políticas y los planes de inclusión. El empleo sigue siendo a día de hoy la principal preocupación de la ciudadanía, y por tanto, debe ser un objetivo prioritario, pero siempre que sea una meta alcanzable para la persona. Equivocadamente, hemos entendido que todas las personas en situación de exclusión debían aspirar a conseguir este objetivo y hemos ido creando, para ello, sistemas protegidos de empleo.

La búsqueda persistente de este objetivo para todas las personas usuarias de los servicios de inserción ha supuesto, en muchas de ellas, una gran frustración, al resultar imposible de alcanzar. Además, indirectamente las hemos culpabilizado por ello, sin ser conscientes de que este fracaso tenía más que ver con la propuesta de un objetivo equivocado que con su propia acción.

Debemos creer que la inclusión de las personas con baja empleabilidad es posible y seguir trabajando por conseguir su verdadera integración al margen del empleo, transcendiendo así el concepto de inserción como incorporación laboral.

\subsection{Lanzamiento de un mensaje equivocado a la sociedad}

El contexto social actual es más individualista que el del inicio de la democracia. Entendemos que aportamos al bien común a través del pago de impuestos, que participamos a través del voto, y delegamos nuestra responsabilidad como ciudadanos en las administraciones públicas. Las entidades del tercer sector hemos contribuido a reforzar este esquema de pensamiento, según el cual quien no trabaja no aporta, y quien no aporta no puede llegar a estar, ni sentirse, socialmente integrado. Ciertamente, se trata de personas que tienen sus derechos, pero no pueden hacerse cargo de sus deberes. Se ha generado una sensación de que las personas en exclusión deben ser apoyadas por la Administración, convirtiéndose así en una carga para la sociedad y en una especie de ciudadanía de segunda. Ello conlleva que las personas empiecen a ser percibidas como incómodas y potencialmente peligrosas.

Para corregir esta corriente, debemos abordar la sensibilización desde otras claves, empezando por no sobredimensionar la idea de que la sociedad debe ser solidaria con aquellos que peor están. El ciudadano medio ya lo sabe, y acepta que hay que apoyar a los más débiles. Las personas en situación de exclusión deben tener contacto con el resto de la 
sociedad en contextos normalizados, en los que se mueven el resto de personas. Tememos aquello que no conocemos, y por eso el contacto debe ser, en la medida de lo posible, cercano, persona a persona.

Es necesario buscar modos de participación a través de los cuales las personas en exclusión aporten valor, presentándose no como personas con problemas, sino como ciudadanas que hacen algo útil para el entorno comunitario en el que están. Esto refuerza la idea de que se puede estar incluido sin tener un empleo remunerado. La inclusión no se puede valorar en función de lo cerca o lejos que se está de un trabajo y de un funcionamiento autónomo. Lo aceptamos con normalidad en muchos casos: personas jubiladas, estudiantes, personas que se encargan de gestionar su hogar. Ahora también, en personas con discapacidades. Debemos conseguir aceptarlo en más grupos de personas.

\section{La oportunidad de descubrir nuevos modos de inclusión social}

Esta constatación de pérdida de algunas claves importantes nos sitúa en un escenario de búsqueda, de oportunidad de descubrir nuevos modos de inclusión social, y para ello disponemos de elementos sobre los que podemos apoyarnos.

\subsection{Inclusión, vida independiente y diversidad funcional}

Durante estos últimos años, ciertos colectivos nos han ayudado a dar la vuelta a algunas situaciones. Han trabajado la sensibilización social desde el enfoque de los derechos, consiguiendo un nuevo modelo. La idea básica de fondo es que, allá donde no pueden llegar las personas, la sociedad se tiene que adaptar, generando sistemas que faciliten el acceso, si es necesario, de manera permanente y generalizada. La lucha exigente en clave de derechos de las entidades de apoyo a la discapacidad física, por ejemplo, ha conseguido cosas que hace pocos años eran impensables. Socialmente tenemos asumido que no se puede forzar a las personas a que hagan aquello que les resulta imposible, y que las personas con dificultades de movilidad, como ciudadanos de pleno derecho que son, deben disponer de sistemas que faciliten la accesibilidad a lo largo de toda su vida.

Éste es el camino a seguir desde el mundo de la exclusión. Donde hay personas que no pueden acceder al empleo remunerado y a la autonomía funcional plena, debemos, desde el enfoque del derecho, poner sistemas de apoyo permanentes que les permitan hacerlo. Así, deberíamos ser capaces de extender a las personas en situación de exclusión social los postulados y objetivos de los conceptos de vida independiente y diversidad funcional. Es verdad que estos sistemas de apoyo requieren de imaginación: primero, porque hace falta un cambio de mentalidad social que comprenda que alguien que no trabaja y no es autónomo puede ser una persona incluida; y segundo, porque para estar (y sentirse) incluido, es indispensable tener un papel activo en la sociedad, aportando al bien común. Para conseguir esto, personas que no son autónomas en su desempeño diario van a necesitar apoyos durante toda su vida, y esto va a requerir respuestas novedosas.

\subsection{Aportación social y trabajo productivo}

Vivimos en una sociedad en la que el intercambio de valor se mide fundamentalmente en términos monetarios. Por ello, cuando pensamos en lo que las personas podemos aportar entendemos, automáticamente, que el desempeño de un trabajo productivo es la mejor manera de contribuir al bien común. Producimos un valor, en forma de bien o servicio, por el que recibimos un sueldo, y parte de dicho salario lo aportamos a las administraciones públicas, que a su vez nos lo devuelven en forma, normalmente, de servicio.

Aceptamos el trabajo reproductivo como parte de la aportación social. A través de él, nacen y se desarrollan personas que van a acabar realizando un trabajo productivo. También nos parece correcto que personas que han pagado impuestos derivados del empleo a lo largo de su vida reciban una asignación mensual cuando dejan de hacerlo: temporalmente, quienes caen de baja o se encuentran en paro; y de manera permanente, aquellas que se jubilan o sufren una enfermedad discapacitante.

Sin embargo, nos cuesta entender como aportación otras tareas que no se asocian con un intercambio económico, bien directo, bien diferido. Debemos ampliar nuestras miras y hacer valer otras aportaciones sociales. Sería una especie de trabajo productivo de no mercado, que ofrece otro tipo de servicios a la comunidad. Y lo hace en los contextos cercanos de convivencia, como el barrio.

\subsection{Los vínculos comunitarios}

El psicólogo Urie Bronfenbrenner (1987), en su teoría ecológica del desarrollo humano, definió que hay diferentes contextos que son importantes en la conformación de la personalidad. Son una serie de sistemas que se superponen, y que van desde el ontosistema (las características propias de cada individuo) hasta el globosistema. A lo largo de nuestra vida nos vemos constantemente afectadas por las interacciones que se producen en estos sistemas (Cuadro 1).

Los cambios sociales de estas últimas décadas han generado un cambio en la influencia de cada uno de estos contextos sobre nuestra vida. Destacamos, de manera sintética, dos fenómenos:

- La globalización. La mejora de los sistemas de comunicación y su accesibilidad para la 


\begin{tabular}{|l|l|}
\hline Sistemas & Interacciones \\
\hline Microsistemas & Incluyen las interacciones personales más cercanas: familia, grupo de amigos. \\
\hline Mesosistemas & $\begin{array}{l}\text { Son aquellos que se forman cuando dos o más microsistemas interactúan entre sí, con el individuo como punto } \\
\text { central común de dichas relaciones. }\end{array}$ \\
\hline Exosistemas & $\begin{array}{l}\text { Son más ambientales. Afectan a la persona, aunque ésta puede no participar directamente. Son el barrio, la } \\
\text { localidad. }\end{array}$ \\
\hline Macrosistemas & $\begin{array}{l}\text { Son los marcos ideológicos o culturales que afectan a los sistemas menores. Configuran los valores de la } \\
\text { sociedad, los modelos económicos, los condicionamientos sociales. }\end{array}$ \\
\hline
\end{tabular}

Fuente: Elaboración propia a partir de Bronfenbrenner (1987).

ciudadanía ha generado que eventos que suceden a muchos kilómetros de distancia puedan estar muy presentes en nuestras vidas casi al momento. Hoy en día, las nuevas tendencias y los cambios sociales pueden extenderse prácticamente a escala mundial en un espacio muy corto de tiempo. Esto hace que los sistemas intermedios pierdan su importancia como vehículos que facilitan la transmisión de tendencias que se producen en otros lugares y como elemento de identificación de la persona, porque nos podemos sentir parte de algo más global sin necesidad de relacionarnos con estas estructuras de menor tamaño.

- El individualismo. Vivimos más hacia adentro de lo que vivían nuestros padres. Ya no necesitamos de nuestro entorno comunitario para solucionar algunos de nuestros problemas. Cuando no los podemos resolver personalmente, esperamos que sea el Estado, a través de las administraciones públicas, quien se haga cargo. Esto nos lleva a relacionarnos mucho menos con personas que no forman parte de nuestros círculos más íntimos. El ejercicio de nuestros deberes, y el disfrute de nuestros derechos de ciudadanía, no necesita de la participación en entornos intermedios. Pierden fuerza, por tanto, las dinámicas comunitarias y pierden oportunidades de participar aquellas personas que no tienen la fuerza suficiente para hacerlo por sí mismas y las que no cuentan con microsistemas fuertes que les permitan compensar sus debilidades personales.

Así, los exosistemas más cercanos al individuo (el entorno cercano, el barrio) han ido perdiendo importancia en la vida de las personas. Hemos creado una sociedad que no necesita que sus miembros interactúen entre sí para formar parte de ella. Una sociedad que puede seguir caminando sin una de sus bases fundamentales: las relaciones. Es importante que trabajemos para recuperar su importancia: primero porque son fundamentales en toda sociedad sana; y sobre todo, porque son indispensables para que puedan formar parte de ella muchas de las personas que se encuentran en situación de exclusión social.

\section{Evolución del concepto de la activación}

Por lo explicado anteriormente, la exclusión social no podemos entenderla como la distancia que separa a la persona del mundo laboral y de la autonomía en su funcionamiento y en la toma de decisiones. Llevamos muchos años desarrollando y utilizando herramientas que nos sirven tanto para valorar las situaciones de exclusión como para diseñar planes de acción orientados a corregirlas. La más completa es la elaborada por el Gobierno Vasco (2012b). Su base es un modelo de diagnóstico social según el cual "para poder determinar en qué situación se encuentra una persona, se ofrece un total de 171 indicadores, ordenados por dimensiones $y$, a su vez, organizados en 5 ámbitos vitales: económico, convivencial, personal, salud y social” (ibídem: 3).

El empleo, de manera directa, produce mejoras sólo en algunos de estos indicadores, aunque, de manera indirecta, puede repercutir en muchos más. Sin embargo, para que lo haga deben darse otra serie de condicionantes tanto personales como del contexto social y del entorno de residencia. Con todo, lo cierto es que el empleo sigue siendo el objetivo principal de muchas de las políticas de inclusión social. Ello convierte en especiales o excepcionales las situaciones que viven aquellas personas en exclusión con dificultades importantes de acceso al mercado laboral, si bien los planteamientos en torno a esta cuestión han ido cambiando estos últimos años.

\subsection{El enfoque de la inclusión activa}

El III Plan Vasco de Inclusión Activa (2012-2016), elaborado por el Gobierno Vasco (2012a), parte del doble derecho reconocido en la Ley de Servicios Sociales (12/2008): a unos ingresos mínimos y a la inclusión social. Su objetivo es: "promover la construcción de una sociedad en la que todas las personas que la conforman se sientan con los mismos derechos y obligaciones, al margen de su situación económica y social, en la que sus diferencias sean respetadas y sus necesidades básicas se encuentren cubiertas" (ibídem: 12). Este 
objetivo lo concreta definiendo una perspectiva que garantice "un nivel de ingresos mínimos adecuados ligados a la activación, el apoyo al acceso a un empleo decente, $y$ a unos servicios públicos que faciliten los medios adecuados para apoyar la autonomía personal de las ciudadanas y los ciudadanos en todos los ámbitos de su vida" (ibídem: 13).

La inclusión activa sigue poniendo el empleo en el centro. Entiende que todas las personas "son empleables, o pueden mejorar su empleabilidad" (loc. cit.). Sin embargo, establece excepciones: "los pensionistas, la invalidez absoluta, personas afectadas por enfermedades y trastornos psíquicos graves invalidantes, las personas menores de 23 años que cursen estudios reglados, y las personas en situación de alta exclusión que, a juicio del profesional correspondiente, no se encuentren en situación de incorporarse al mercado laboral" (loc. cit.). Por otro lado, parte del principio de reciprocidad entre la Administración y la ciudadanía, y se operativiza a través de un obligado convenio de inclusión, por el que la persona se compromete a dar una serie de pasos encaminados a su plena incorporación social.

Pero este enfoque, que en su día fue un gran avance, presenta algunos problemas, siendo el primero que el concepto de reciprocidad está asociado al empleo. El trabajo lo entendemos como algo muy ligado al intercambio económico, y ello deriva en una interpretación reduccionista del término, que nos puede llevar a pensar que la única forma de devolver a la sociedad lo que ésta nos da es a través del dinero.

En segundo lugar, establecer que la solidaridad se produce entre la Administración y el ciudadano puede llevar a confundir el todo con la parte. Una sociedad sólo puede existir cuando existe un sistema de solidaridad mutua entre sus miembros que hace que, juntos, seamos más fuertes que individualmente. Este sistema de apoyos se da entre todas las personas: no se trata sólo de que las fuertes apoyan a las débiles, sino de que cada una de ellas pone sus fortalezas al servicio del bien común. Las sociedades complejas, como la nuestra, necesitan crear entidades que gestionen todo este entramado de derechos y obligaciones, y por ello existe la Administración. Ésta es sólo un instrumento que nos permite gestionar la solidaridad entre las personas. Por lo tanto, convertirla en sujeto de dicha solidaridad nos lleva a confusiones sobre su papel y sobre el nuestro propio. Estos equívocos son perjudiciales, especialmente para las personas en situación de exclusión.

Por último y volviendo sobre nuestra preocupación principal, está la centralidad del empleo. Es verdad que, en una sociedad compleja, es el modo más útil y debe ser el más común para desarrollar esta reciprocidad. Sin embargo, entenderla como la única herramienta nos lleva a convertir a las personas no empleables en excepciones, y esto es un error. Cierto es que son una minoría, porque no pueden utilizar el mecanismo de aportación más común, pero entenderlas como una excepción aumenta el estigma sobre ellas y disminuye sus posibilidades de participación.

\subsection{Activación inclusiva y ciudadanía activa}

Los conceptos de activación inclusiva y ciudadanía activa suponen dan un paso más. Aunque reconocen que el empleo es un factor esencial en la inclusión social, admiten que, por sí mismo, no la garantiza. Adoptan, por lo tanto, el principio de reciprocidad justa, que implica "no ignorar o privilegiar arbitrariamente ciertas formas de contribución social sobre otras, evitando equiparar de forma reduccionista el concepto de contribución productiva con el de empleo remunerado" (SIIS Centro de Documentación y Estudios, 2012: 118). Valora, por lo tanto, otros tipos de aportación social relacionadas con la participación, especialmente en actividades de cuidados familiares, acción comunitaria, relaciones sociales y ocio.

Ekain (2016), plataforma que engloba a cinco redes de intervención social no lucrativa de Euskadi, plantea, dentro de este concepto, el desarrollo de estrategias de inclusión desde tres claves:

- Reconocer que las personas pueden aportar a la comunidad de diferentes maneras, no sólo desde el empleo: desde el voluntariado, desde la ciudadanía activa, desde la participación en la vida socio-comunitaria. Tratar a las personas y ver cómo se garantizan sus derechos (y deberes), por el mero hecho de ser ciudadanos y ciudadanas, al margen de su situación laboral.

- Facilitar procesos de inclusión desde el desarrollo comunitario; además de garantizar a las personas las condiciones básicas para una vida (alimentación, alojamiento, vestido...), favorecer la participación de la persona en la vida comunitaria, facilitar la interrelación con la comunidad, con el vecindario... Vincular a las personas con su entorno.

- Enfocar el trabajo en los servicios desde la perspectiva del trabajo centrado en la persona.

El enfoque de la activación inclusiva presenta algunos avances sobre el anterior. El más importante es que introduce con fuerza elementos de aportación social diferentes al del empleo, abriendo así oportunidades de participación activa a las personas que no pueden acceder a aquél. Por otro lado, el cambio de orden de las palabras hace que pongamos el foco más en la activación que en la inclusión. Es, por tanto, un punto de partida más positivo: considerar a las personas como activables, antes que como excluidas, mejora su consideración social y, por lo tanto, sus opciones de inclusión. 
Una debilidad de este concepto es que está centrado en la acción individual, y la realidad nos dice que las personas en situación de exclusión que están más lejos del empleo también lo están de otros modos de participación y necesitan estar integradas en el marco de acciones colectivas para poder aportar socialmente.

\subsection{Activación sociocomunitaria}

Desde Bizitegi, proponemos un enfoque que facilite la creación de sistemas que posibiliten acompañar más a las personas en su participación. Que permitan integrar a aquellas que, aun teniendo dificultades de relación o falta de autonomía, cuentan con plena capacidad para aportar. Para ello, lo primero que tenemos que hacer las entidades que apoyamos a personas en exclusión es posicionarnos en el entorno $y$ hacerlo de un modo diferente al que estamos acostumbradas. Nuestro proceder habitual a la hora de colaborar con otros elementos de la sociedad es el de crear redes, con entidades o personas afines, partiendo de un objetivo común previamente establecido.

Sin embargo, tal y como postula Daniel de Torres (2017), en las comunidades no creamos redes, sino que establecemos relaciones. Por ello, el primer objetivo de nuestras organizaciones debe ser interactuar con el barrio, con el único objetivo de construir relaciones de cohesión con éste. Conocer el entorno, a las personas, las organizaciones vecinales. Convivir con ellas, saber sus inquietudes, sus problemas y sus anhelos, sin más pretensión que la de ser un sujeto más. Un sujeto que forma parte de las iniciativas que se generan, que contribuye a promoverlas, que busca soluciones y aporta como uno más, que se preocupa por los problemas del barrio, que es capaz de tomar la bandera de la comunidad como propia, dejando de lado la de la exclusión.

Es desde este planteamiento desde el que podemos hacer que las personas que están excluidas participen. Con esas relaciones ya creadas, podemos ir incorporándolas. Es importante que su rol no sea el de personas que se encuentran en situación de exclusión: ellas también tienen que dejar esa bandera de lado. Son participantes, unas vecinas más, que aportan mediante tareas que en ese contexto ya están aceptadas como útiles. Dichas aportaciones pueden ser tanto individuales como grupales. Es responsabilidad de las organizaciones buscar oportunidades de participación en las que quepan todas. Muy pocas personas se encuentran en un momento tan frágil como para no poder ofrecer nada al bien común.

Todo esto, además, tiene un efecto positivo sobre la ciudadanía, ya que conlleva una sensibilización no basada en campañas ni el despertar del sentimiento de solidaridad de los otros, sino que se apoya en la convivencia y el conocimiento mutuo. Va a las bases mismas de las relaciones humanas y al reconocimiento entre iguales, puesto que implica conocerse de tú a tú, obviando el rol social de la persona en exclusión, dejando que desempeñe un papel activo, que desarrolle una tarea que resulta útil al entorno. Al ser éste un papel que contrasta con 'lo esperado', descoloca a las personas que están en frente, y las obliga a adoptar un contra-rol diferente al habitual, una postura de igual a igual. Por lo tanto, no cambia sólo pensamientos, sino que altera la propia posición de quien se encuentra al lado.

En resumen, si nos esforzamos de manera genuina por fortalecer la comunidad y crear cohesión, creamos las condiciones para que las personas que más difícil lo tienen puedan alcanzar sus mayores cotas de integración, consiguiendo así el objetivo que propone Daniel de Torres (2017) de integrar lo comunitario y lo asistencial.

\section{Marcos de referencia}

Nuestra propuesta va en línea de un cambio de paradigma, una evolución del modelo de servicios sociales. Para ello, conecta con las preocupaciones y propuestas de diversos autores que nos ayudan a conformar el proyecto. Así, yendo de lo más general a lo más concreto, presentamos de manera sintética una serie de marcos de referencia que, bien por haber acudido a ellos como referencias teóricas o por haber incluso participado activamente en su desarrollo, nos permiten situar el proyecto de activación sociocomunitaria dentro de una tendencia de cambio de orientación de los servicios sociales tanto en su objeto como en su concepción.

\subsection{Definición de riqueza y progreso}

Esta propuesta conecta con la preocupación existente sobre la necesidad de desarrollar indicadores de calidad que midan el comportamiento de la sociedad en los ámbitos que reflejen su progreso social. Superar la medición del bienestar de una sociedad y de sus miembros únicamente a través de medidores económicos. La medición del bienestar tiene que tener necesariamente un componente de riqueza material, pero debe complementarse con indicadores que tengan en cuenta otros factores de calidad de vida, que incluyan aspectos relacionales y de cuidado que forman parte del ámbito de 'actividades de no mercado'.

La necesaria y conveniente participación de las personas en el esfuerzo económico y productivo como medio de participación y de aportación social, a la vez que como medio de acceder a un adecuado reparto de la riqueza generada, si bien debe tener un carácter predominante en una sociedad que desee ser próspera, no puede tener un carácter exclusivo en una sociedad que desee ser justa. Este enfoque economicista tan limitado que reduce la consideración de bienestar social a indicadores como el PIB es germen de exclusión social, ya que, 
de manera derivada, sólo concede valor y utilidad a los trabajos, y por tanto a las personas que pueden desempeñarlos en situación de mercado competitivo. Las personas que, por su situación e historia, tienen dificultades de acceso a puestos de trabajo en esas condiciones parecen quedar fuera del círculo de aportación útil a la sociedad.

Informes como el elaborado por la comisión StiglitzSen-Fitoussi (2009) abren el horizonte a considerar a estas personas no solamente como sujetos de derechos sociales en consideración a los valores de justicia social y solidaridad, sino que también abren la puerta a que se puedan motivar y realizar actividades de 'no mercado', pero que tengan una repercusión palpable y valorable en el bienestar de las comunidades desde donde se ejerce y favorece, por tanto, el progreso social.

\subsection{Binomio sociedad-comunidad}

En un plano general, Xabier Aierdi (2014) nos plantea las contradicciones que surgen en el momento histórico actual entre sociedad y comunidad. Nos lanza unas cuestiones a modo de reto que necesariamente debemos tomar en cuenta a la hora de plantear cualquier propuesta de trabajo con la comunidad, unas propuetas que, lejos de presupuestos idealistas, nos tienen que enfrentar a la realidad de una sociedad y una ciudadanía con mayores niveles de individuación y con un debilitamiento claro de los referentes religiosos e ideológicos que facilitaban anteriormente establecer y compartir un imperativo moral. En este horizonte de "fatiga de la solidaridad", hay que avanzar por vías de utilidad y bienestar social.

\subsection{Relaciones de cohesión}

Siguiendo a Marchioni (2001), el proceso de individuación nos ha llevado a crear también una estructura de servicios y prestaciones en torno a la intervención individualizada, pero es preciso pasar a una intervención comunitaria. Esto supone no solamente una extensión del campo de acción, sino un cambio total de perspectiva, ya que pasamos a un proceso de construcción de relaciones de cohesión que no ofrece prestaciones de manera directa. Nos situamos además frente a un proceso sin final, ya que el proceso de construcción comunitaria no termina, al tener que adaptarse constantemente a los cambios sociales. Por otro lado, no hay que caer en la contraposición entre trabajo comunitario y asistencial (De Torres 2017), sino que ambos enfoques deben integrarse partiendo de un reconocimiento de la aportación social de las personas usuarias.

\subsection{Objeto propio de los servicios sociales}

Fernando Fantova (2017) plantea una búsqueda del objeto propio de los servicios sociales en un contexto de paso de una asistencia social residual no especializada a unos servicios sociales universales que deben establecer a qué necesidades deben dedicarse. El desarrollo de los diversos sistemas públicos de provisión de servicios educativos, sanitarios, de vivienda y de recursos económicos debe llevar a una especialización de los servicios sociales en aquello que en ningún caso va a ser cubierto por esos sistemas y que constituye la expresión mayor de la exclusión: la ausencia de relaciones sociales. En este sentido, su propuesta aboga por considerar la interacción -entendida como el desenvolvimiento autónomo de las personas en su vida diaria en el seno de relaciones familiares y comunitarias - como el bien que protege y promueve los servicios sociales.

El proceso de construcción de una atención integrada, entendida como cadenas de valor que posibiliten itinerarios de consecución de resultados valiosos para las personas, introduce un nuevo elemento de innovación, al planear la atención y los servicios desde una óptica desde la cual la ubicación de la sede física no es relevante, y al abrir las puertas a programas virtuales y comunitarios. Esta nueva concepción también permite una mejor gestión de la diversidad y de los avances tecnológicos.

\subsection{Pedagogía social comunitaria}

La pedagogía social comunitaria presentada por Txus Morata (2014) plantea un modelo de intervención socioeducativa que combina los objetivos de mejorar la calidad de vida de las personas y trabajar por la cohesión social. Este modelo, que entronca con la trayectoria de participación comunitaria de Bizitegi, nos sirve de base de trabajo.

Como punto de partida, es preciso entender el bienestar subjetivo como el resultado del balance global que hace la persona de sus oportunidades vitales, del curso de los acontecimientos a los que se enfrenta y de la experiencia emocional que se deriva de ello. Sin embargo debemos ir dando pasos hacia el empoderamiento, entendido como el proceso mediante el cual las personas ganan control y poder sobre sus vidas. Un empoderamiento que pasa del plano individual al comunitario. Un empoderamiento que, a veces incluso, resulta incómodo para los profesionales, por conceder gran valor a la opinión de la persona usuaria.

Este modelo plantea tres objetivos prioritarios: la participación y la construcción de ciudadanía como elementos clave para luchar contra la exclusión, la corresponsabilidad como red que genera ciudadanía, y la sensibilización y el cambio social. Estos objetivos se concretan en tres metodologías de trabajo: el acompañamiento psicosocial, la animación sociocultural y el trabajo comunitario. 


\section{Una posible propuesta: Aurkigune}

\subsection{Planteamiento}

Siguiendo los principios desarrollados en este artículo y con objeto de superar un esquema meramente teórico y dar pasos en su materialización práctica, creemos que podría ser útil la propuesta de creación de un centro, Aurkigune, que plantee itinerarios de inserción social distintos del empleo y que busque el desarrollo personal de las personas implicadas a través de su aportación de valor a la comunidad desde sus potencialidades, generando un efecto positivo en la ciudadanía, que suscite un cambio de percepción social sobre estos colectivos.

Este proyecto se sustancia en la creación de un centro comunitario de activación social que conecte colectivos vulnerables con el barrio, para así aumentar su inclusión social mediante:

- El refuerzo de las capacidades de las personas, para revertirlas en el barrio y así desestigmatizar al colectivo

- La detección de necesidades de la comunidad que permitan generar actividades de 'no mercado' adaptadas a las personas con discapacidad y en situación de exclusión.

- El fomento de la solidaridad de las personas a través de acciones de voluntariado.

El colectivo al que va dirigido es el de las personas en situación de exclusión social con difícil empleabilidad. También será beneficiaria del centro la comunidad en la que se desarrolle.

El objetivo de este centro de activación sociocomunitaria sería la participación de personas con graves dificultades y escasas posibilidades de inclusión laboral en actividades denominadas de "no mercado', que permitan una doble contribución al bienestar: a través de la participación normalizada de la persona que presta su tiempo y a través de la persona que recibe la atención. Pretende ser punto de encuentro entre la voluntad de las personas que voluntariamente deseen dedicar parte de su tiempo y de las personas o entidades sociales que necesiten de esa dedicación.

El cambio de enfoque que supone adoptar la perspectiva de las personas con mayores dificultades es radical, ya que permite trabajar desde la óptica de la inclusión social y relacional, y simultáneamente, desde la óptica de la aportación a la sociedad. A la vez, refuerza la capacidad de decisión de estas personas sobre el destino de su tiempo y les convierte, no solamente en sujetos de derechos, sino también en fuente de solidaridad.

Conjugar las perspectivas del derecho subjetivo a la inclusión social, de la solidaridad en la dedicación, con la contribución al progreso social hace de este proyecto un hito que puede suponer el inicio de un recorrido que, enmarcado en un enfoque comunitario, permita un avance en el bienestar de las personas que participen en él.

\subsection{Funciones principales}

Las tareas y funciones principales de un dispositivo de estas características son las siguientes:

- Identificación de necesidades en la comunidad. El primer paso es conseguir formar parte del barrio; unirse, junto con otros agentes, a iniciativas vecinales encaminadas a mejorar el entorno. La pertenencia a estas plataformas permite identificar necesidades no cubiertas. Es muy importante que la participación de Aurkigune en estos entornos sea proactiva, que ayude a detectar y poner nombre a las posibilidades de apoyo que puedan surgir. Una vez conocido el proyecto por la comunidad, es posible intensificar la búsqueda de oportunidades, registrando las necesidades detectadas por las personas a través de encuestas individuales y grupales que se entregarán en cada visita y entrevista individual o grupal.

Se puede impartir formación a las personas y comercios interesados, sobre atención y trato a personas vulnerables. Esta formación será impartida por las propias personas participantes del proyecto, con el fin de romper con el estigma de estos colectivos y poner en práctica los contenidos de los talleres de competencias.

Es clave la coordinación continua con los agentes comunitarios implicados de forma telefónica y presencial, registrando su grado de satisfacción en las colaboraciones dadas.

- Selección de las personas participantes. Se realiza a través de los servicios sociales de base, los centros colaboradores de orientación de Lanbide para colectivos en desventaja y los servicios forales de atención tanto de personas en situación de exclusión social como de personas con discapacidad. Sería interesante que estas personas cumplieran dos o más de los siguientes requisitos:

- Tener el reconocimiento de colectivo en desventaja según el servicio social de base: ser perceptoras de la renta de garantía de ingresos, tener reconocida una discapacidad del $33 \%$ o superior, estar en proceso de rehabilitación por drogodependencias o estar consideradas por Lanbide como personas con perfil de empleabilidad bajo, por ejemplo.

- Estar empadronadas en la localidad de ubicación.

Además, estas personas deberán estar informadas de sus derechos, obligaciones y del beneficio de participar en este programa, y se firmará un compromiso de participación voluntaria. 
- Información, formación y capacitación de las personas participantes. Desde el inicio del proyecto es importante incidir en el empoderamiento de estas personas, dándoles la oportunidad de presentarse al barrio como promotores de la iniciativa, dinamizando grupos que versen sobre los valores y la filosofía del proyecto, de tal modo que los participantes lo sientan como algo propio que ellos mismos pueden construir.

Se realizará un diagnóstico y se procederá a la elaboración del perfil de participantes. Ello comprenderá todas las acciones dirigidas a identificar necesidades e intereses, a detectar habilidades, potencialidades y apoyos requeridos. Una vez identificados los perfiles, se emparejarán con las necesidades de la comunidad que se pretende cubrir (ya sea de forma individual o en grupo).

Es importante también llevar a cabo acciones formativas para fomentar la participación. Deben realizarse en grupos conformados en función de los perfiles detectados e incidir especialmente en el desarrollo de capacidades y habilidades para la participación, el fomento de la participación activa y responsable de las personas, la detección de personas que puedan liderar procesos de empoderamiento individuales y grupales, y la motivación de las personas usuarias para la participación social.

- Diseño del plan de activación. En esta fase, se contempla la disponibilidad de la persona, la tarea o tareas a realizar, el modo de realización, los apoyos requeridos, la duración del plan, así como la supervisión y evaluación de los compromisos adquiridos.

- Puesta en marcha y seguimiento de las acciones. Pueden ser tanto individuales como colectivas, en función tanto de la necesidad detectada, como de las posibilidades de ofrecer apoyo de cada persona participante. La realización de las acciones diseñadas implica un acompañamiento individual y grupal de la puesta en marcha de los planes, un control de incidencias, registros de planes, realización de encuestas, y cambios y modificaciones en tareas, entre otras cuestiones.

- Gestión del centro. Las personas participantes deben ser las encargadas de la apertura del centro, la acogida a las personas que se acerquen, el registro de asistentes, la actualización de noticias, la revisión de tablones o la atención telefónica. Hay comisiones de trabajo que se organizan para realizar esas funciones. Las asambleas periódicas funcionan como espacio de participación, donde las personas hablan de cuestiones relacionadas con sus tareas y con nuevas ideas a desarrollar en el entorno; en ellas, podrán formarse subgrupos o comisiones de trabajo que dinamicen actividades concretas. Las asambleas son también un espacio de resolución de conflictos, donde el grupo puede aportar vías de solución a los problemas. Finalmente, son un espacio donde cada persona asume un rol activo, incluido el del liderazgo de las propias reuniones, que es rotatorio.

\subsection{Factores de éxito}

Hay una serie de factores que son claves para el éxito de un dispositivo de estas características:

- La conexión con el entorno comunitario y la socialización del proyecto. Garantizar la máxima difusión y hacer que llegue a todos los miembros de la comunidad permite que las personas sientan que se las tiene en cuenta y que se las quiere hacer partícipes de un proyecto conjunto. Desde la cercanía y apertura, y con afán de construir una comunidad más inclusiva, nos acercaremos a personas y organismos del entorno, socializando la iniciativa y recogiendo opiniones, reflexiones y aportaciones que puedan enriquecer el proyecto.

- La identificación de necesidades. Identificar necesidades con relevancia para la o las personas a las que se les va a dar cobertura es un factor importante. Conviene utilizar diferentes metodologías (entrevistas, cuestionarios, World Café) para detectar necesidades y analizar tareas. Merece la pena tratar de realizar un buen análisis de las tareas, identificando el mayor número posibles de variables que incidan en ellas.

- El ajuste entre las tareas y las competencias de la persona que las presta. Es esencial definir un buen perfil personal del colaborador de la tarea, para así garantizar la satisfacción de la persona que necesita el servicio. Este buen ajuste dependerá de la formación previa, la complejidad y la dedicación que requiere, el número de personas que interactúan en su ejecución, el contexto y el espacio donde se realice, y de la buena identificación de la necesidad y del análisis pormenorizado de las tareas que componen esa necesidad.

- La percepción de utilidad del centro por parte del entorno. Es importante que las actividades en la comunidad se ejecuten con éxito y se hayan difundido convenientemente en el entorno. En la medida en que las personas se impliquen en las actividades del barrio y se las conozca por su contribución, la visión sobre este colectivo se irá ampliando, y será más aceptada y reconocida. De este modo, el centro se irá haciendo hueco en el entorno comunitario. 


\section{Bibliografía referenciada}

AIERDI, X. (2014): "Los nuevos dilemas del binomio sociedad-comunidad" [presentación], en XX Semana Europea de la Gestión Avanzada: 'Innovación social. Participación Comunitaria', Bilbao, Bizitegi.

BRONFENBRENNER, U. (1987): La ecología del desarrollo humano. Experimentos en entornos naturales y diseñados, Barcelona, Paidós.

DE TORRES, D. (2017): “Construyendo barrio a través del intercambio de experiencias", en Jornadas de Intervención Comunitaria: intercambiando experiencias europeas, estatales y locales, Bilbao, Médicos del Mundo

EKAIN-REDES PARA LA INCLUSIÓN EN EUSKADI (2016): 57 Propuestas de las Redes para la Inclusión Social en Euskadi a los partidos políticos para las elecciones al Parlamento Vasco 2012, Bilbao, Ekain-Redes para la Inclusión en Euskadi [<http://www.ekainsarea.net/ documentos/propuestas.pdf)].

FANTOVA, F. (2017): "Servicios sociales: objeto propio y atención integrada”, en Llei d'Engel, 18 de abril [rhttp://lleiengel.cat/serveis-socials-objecte〉].

GOBIERNO VASCO (2012a): III Plan de Inclusión Activa (2012-2016), Vitoria-Gasteiz, Servicio Central de Publicaciones del Gobierno Vasco [rhttp:// www.euskadi.eus/contenidos/informacion/ actuaciones_documentacion/es_documen/ adjuntos/plan_inclusion.pdf`].

- (2012b): Instrumento Técnico Común de Valoración de la Exclusión Social, Vitoria-Gasteiz, Eusko Jaurlaritza-Gobierno Vasco [<http://www. gizartelan.ejgv.euskadi.eus/contenidos/ informacion/instrumento_valoracion/ es_instrume/adjuntos/Modelo $\% 20$ diagnostico\%20social-junio-2012.pdf〉].

MARCHIONI, M. (2001): Comunidad y cambio social: teoría y praxis de la acción comunitaria, Madrid, Edición Popular.
MORATA, T. (2014): “Pedagogía Social Comunitaria: un modelo de intervención socioeducativa integral”, Educación Social. Revista de Intervención Socioeducativa, ํㅡ5ㄱ, págs. 13-32.

PAÍS VASCO (2008): “Ley 12/2008, de 5 de diciembre, de Servicios Sociales", Boletín Oficial del País Vasco, no 246, 24-12-08, págs. 31.840-31.924 [rhttps://www.euskadi.eus/r47-bopvapps/es/ bopv2/datos/2008/12/0807143a.shtml〉].

RAYA LOZANO, E. (2004): Investigar sobre la exclusión para intervenir en lo social. Notas introductorias al Observatorio de Exclusión Social y Políticas de Inclusión de Granada, en RAYA LOZANO, E. (coord.), Exclusión/inclusión social en la ciudad de Granada, Granada, Maristán.

SIIS CENTRO DE DOCUMENTACIÓN Y ESTUDIOS (2012): Activación y derecho a la inclusión en el marco de las políticas de empleo y de garantía de ingresos en la CAPV / Aktibazioa eta gizarteratzako eskubidea, EAEko enpleguko eta diru-sarrerak bermatzeko politiken esparruan, Vitoria-Gasteiz, Eusko Jaurlaritza-Gobierno Vasco [<http://www.siis.net/es/investigacion/ ver-estudio/372/>]

STIGLITZ, J. E.; SEN, A.; y FITOUSSI, J. P. (2009): The Measurement of Economic Performance and Social Progress Revisited: Reflections and Overview, serie Documents de Travail de l'OFCE, no 2009-33, París, Centre de Recherche en Économie de Sciences Po [<https://www.ofce. sciences-po.fr/pdf/dtravail/WP2009-33.pdf)].

SUBIRATS, J. (dir.) (2004): Pobreza y exclusión social. Un análisis de la realidad española y europea, serie Colección Estudios Sociales, $\mathrm{n}$ ำ16, Barcelona, Fundación La Caixa. 